\title{
Soluble Tau has devastating effects on the structural plasticity of hippocampal granule neurons
}

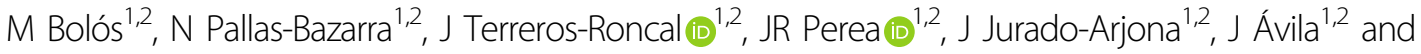 \\ M Llorens-Martín (10 ${ }^{1,2,3}$
}

\begin{abstract}
Tau is a neuronal microtubule-associated protein with countless physiological functions. Although the detrimental effects of insoluble aggregated Tau have been widely studied, recent evidence supports the notion that soluble Tau (composed mostly of monomers and dimers) is also toxic for neurons. Here we evaluated the long-term impact of a single stereotaxic injection of human soluble Tau on hippocampal granule neurons in mice. At the ultrastructural level, soluble Tau reduced the number of afferent synapses and caused a dramatic depletion of synaptic vesicles both in afferent and efferent synapses. Furthermore, the use of an RFP-expressing retrovirus revealed that soluble Tau altered the morphology of newborn granule neurons and reduced their afferent (dendritic spines) and efferent (mossy fiber terminals) connectivity. Finally, soluble Tau caused specific impairment of behavioral pattern separation capacity. Our results thus demonstrate for the first time that soluble Tau causes long-term detrimental effects on the morphology and connectivity of newborn granule neurons and that these effects correlate with impaired behavioral pattern separation skills. These data might be relevant for the field of neurodegenerative disorders, since they contribute to reinforcing the pathological roles played by distinct Tau species in vivo.
\end{abstract}

\section{Introduction}

Tau is a neuronal microtubule-associated protein (MAP) that plays a central role in microtubule stabilization $^{1}$. The relevance of Tau during brain development has been addressed extensively ${ }^{2}$. Moreover, recent evidence points to novel roles played by Tau during adult neurogenesis ${ }^{3}$. Tau regulates not only microtubule stability but also axonal transport ${ }^{4}$ and it plays key roles at synapses ${ }^{5,6}$. During the human lifespan, Tau protein expression and function are intricately regulated by alternative splicing and post-transcriptional modifications ${ }^{7-9}$.

Correspondence: M Llorens-Martín (m.llorens@csic.es)

${ }^{1}$ Department of Molecular Neuropathology, Centro de Biología Molecular

"Severo Ochoa", CBMSO, CSICUAM, Madrid, Spain

${ }^{2}$ Network Center for Biomedical Research on Neurodegenerative Diseases (CIBERNED), Madrid, Spain

Full list of author information is available at the end of the article
A group of neurodegenerative diseases known as Tauopathies is characterized by alterations in Tau metabolism. In these disorders, Tau usually losses solubility and tends to form aggregate structures that impair cell function and trigger neuronal cell death and neurodegeneration $^{9,10}$. Current hypotheses postulate that Tau is released to the extracellular space and propagates from one cell to another following a stereotypic pattern of spread among brain regions ${ }^{11-15}$ and that the presence of this extracellular Tau damages neighboring neuronal and glia cells. It is also believed that the complete aggregation and insolubilization of Tau is not required for this protein to trigger neurodegeneration ${ }^{16}$ and that small oligomers of Tau are highly toxic for neurons ${ }^{13}$. However, the in vivo neurotoxic potential of soluble Tau has not been fully elucidated to date.

The hippocampus is one of the brain regions most affected in Tauopathies ${ }^{17}$. Moreover, synaptic transmission 
to and from the dentate gyrus (DG) is a crucial stage for information processing within the hippocampal trisynaptic circuit ${ }^{18,19}$. Given these considerations, we aimed to explore whether the presence of soluble Tau (composed mostly of monomers and dimers) has long-term effects on the structural plasticity of the predominant neuronal population present in the DG, namely hippocampal granule neurons. Of note, the adult DG has several unique anatomical and functional features. One of the most relevant is adult hippocampal neurogenesis $(\mathrm{AHN})^{20}$, a process that encompasses the generation, maturation, and synaptic integration of newborn granule neurons into the hippocampal trisynaptic circuit. This process is impaired in animal models of Tauopathies and patients with the same conditions, Alzheimer disease (AD) being one ${ }^{21-23}$. These data are in agreement with the relevant role played by these newly generated granule neurons in certain aspects of hippocampal-dependent learning, such as behavioral pattern separation ${ }^{24-26}$. Noteworthy, these cognitive skills are profoundly impaired in patients with Tauopathies ${ }^{27,28}$. In the present study, we show that the stereotaxic injection of soluble Tau into mice causes long-term alterations in the structural plasticity of granule neurons and that these alterations correlate with selective impairment of behavioral pattern separation ability.

\section{Materials and methods Section heading Material and methods changed to Materials and methods, please confirm. OK \\ Animals}

Six-week-old female C57Bl/6J Ola Hsd mice were purchased from EnVigo Laboratories, Spain. Mice were housed in a specific pathogen-free colony facility, in accordance with European Community Guidelines (directive 86/609/EEC), and handled following European and local animal care protocols. Animal experiments received the approval of the CBMSO's (AEEC-CBMSO23/172) and national (PROEX 205/15) Ethics Committee. Animals were left undisturbed for 2 weeks before being subjected to experimental manipulation.

\section{Experimental design}

Animals were randomly assigned to one of two groups, namely PBS-Cy5- or Tau-Cy5-injected. A group of eight mice (4 PBS-Cy5 and 4 Tau-Cy5) was used for retroviral injections (group A). A group of 18 mice (9 PBS-Cy5 and 9 Tau-Cy5) was used for behavioral, histological, and electron microscopy determinations (group B). Of the latter group, three PBS-Cy5- and three Tau-Cy5-injected animals were used for electron microscopy determinations. A schematic experimental design is shown in Fig. 1. Animals were subjected to stereotaxic injections at 2 months of age (red fluorescent protein (RFP)-expressing retrovirus plus either PBS-Cy5 (4) or Tau-Cy5 (4) in the case of group A, and only PBS-Cy5 (9) or Tau-Cy5 (9) in the case of group B). Animals were sacrificed 2 months after stereotaxic injections. Animals belonging to group B received 5-Iodo-2'-deoxyuridine (IdU) at 1 month of age. Behavioral tests were performed on animals belonging to group B immediately before sacrifice.

\section{Tau-Cy5}

The recombinant human Tau isoform containing two $\mathrm{N}$-terminal inserts and four microtubule binding repeats $\left(\right.$ Tau $\left.42^{7}\right)$ was isolated as described previously ${ }^{29}$. Briefly, the proteins isolated were characterized by gel electrophoresis, followed by staining with Coomassie blue. Purified soluble Tau, which is mainly composed of monomers and dimers (Supplementary Figure S1), was labeled with sulfoindocyanine Cy5 dye (GE Healthcare, UK), as described previously ${ }^{30}$ and following the manufacturer's recommendations. Briefly, $1 \mathrm{ml}$ of recombinant Tau protein $(2 \mathrm{mg} / \mathrm{ml})$ was mixed with a sample of Cy5 dye at room temperature for $1 \mathrm{~h}$ to ensure binding to the fluorophore. To remove excess free dye, the mixture was dialyzed overnight at $4^{\circ} \mathrm{C}$ against phosphate-buffered saline (PBS) and filtrated through a Sephadex G-50 column. Cy5-coupled Tau protein was characterized by western blotting with an anti-Cy5 antibody (Abcam; $1: 1000)$.

\section{Ultracentrifugation \\ Sedimentation velocity (SV) assay}

About $0.5 \mathrm{mg} / \mathrm{ml}$ of Tau42 in PBS (pH 7.4) were loaded $(320 \mathrm{ml})$ into analytical ultracentrifugation cells. The experiments were carried out at $48 \mathrm{krpm}$ in an XLI analytical ultracentrifuge (Beckman-Coulter Inc.). Sedimentation coefficient distributions were calculated by least-squares boundary modeling of SV data using the continuous distribution $c(s)$ Lamm equation model as implemented by SEDFIT $15.01 \mathrm{~b}^{31}$. Experimental $s$ values were corrected to standard conditions (water, $20^{\circ} \mathrm{C}$, and infinite dilution) using the program SEDNTERP ${ }^{32}$ to get the corresponding standard sw values $(\mathrm{s} 20, \mathrm{w})$.

\section{Sedimentation equilibrium assay}

Using the same experimental conditions as in the SV experiments, short columns $(90 \mathrm{ml})$ Sedimentation equilibrium experiments were carried out at speeds ranging from 7000 to $10,000 \mathrm{rpm}$. After the last equilibrium scan, a high-speed centrifugation run $(48,000 \mathrm{rpm})$ was done to estimate the corresponding baseline offsets. Weightaverage buoyant molecular weights of protein were determined by fitting a single species model to the experimental data using the HeteroAnalysis program ${ }^{33}$, and corrected for solvent composition and temperature with the program SEDNTERP ${ }^{32}$. 


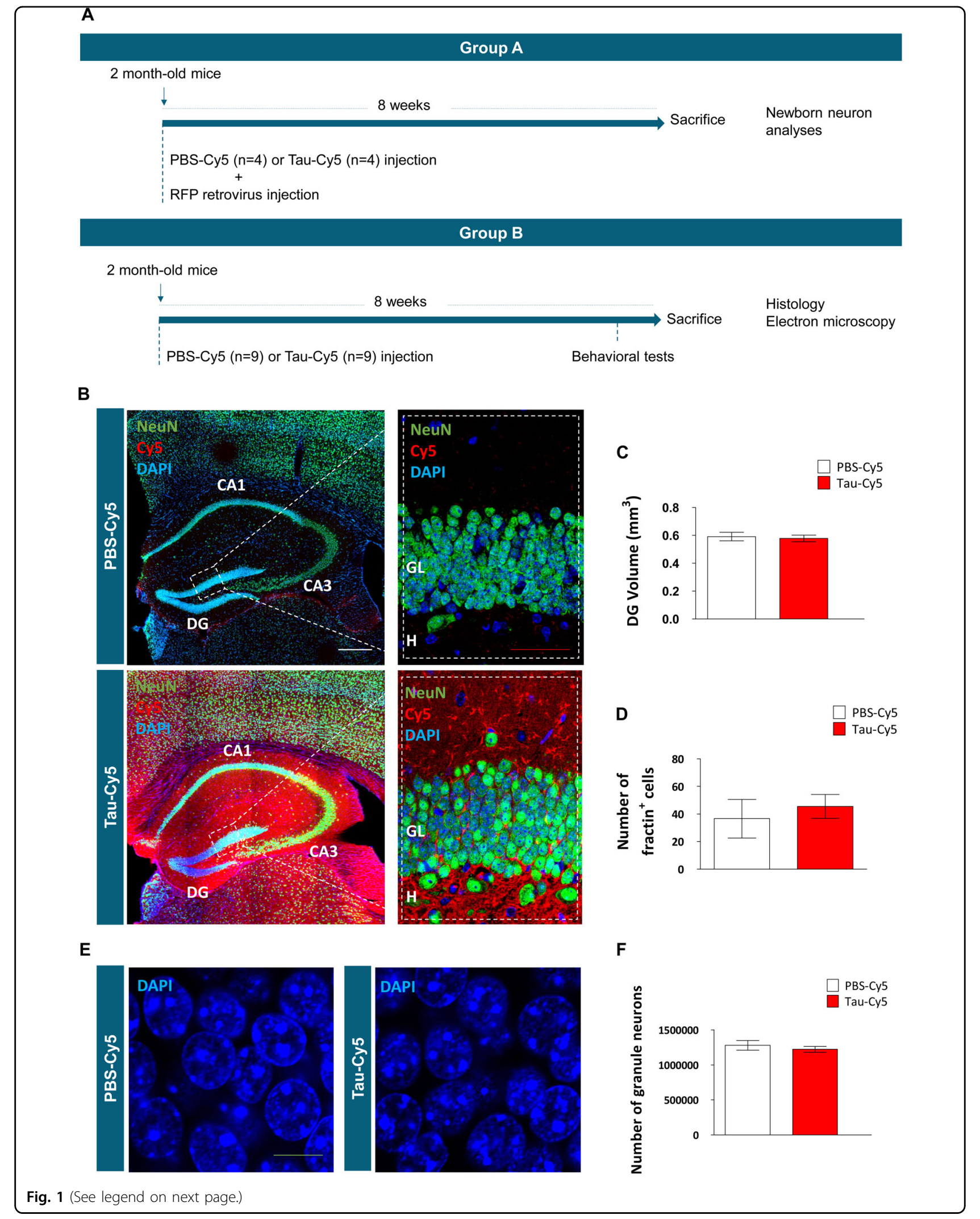


(see figure on previous page)

Fig. 1 The presence of soluble Tau-Cy5 does not alter the gross morphology of the DG. (a) Schematic diagram of the experimental design. Animals received a stereotaxic injection of either Tau-Cy5 (13) or PBS-Cy5 (13) in the hippocampal dentate gyrus (DG). Of these animals, eight (four Tau-Cy5 and four PBS-Cy5) also received an injection of an RFP-expressing retrovirus in order to label newborn granule neurons (group A). The remaining 18 animals (9 Tau-Cy5 and 9 PBS-Cy5) formed the group B. Of these animals, six (three Tau-Cy5 and three PBS-Cy5) were used for electron microscopy analyses. Animals were sacrificed 8 weeks after stereotaxic injections. (b) Representative tile-scan images of the whole hippocampus of PBS-Cy5- and Tau-Cy5-injected mice. An intense Cy5 signal can be observed 8 weeks after Tau-Cy5 injection, but not after PBS-Cy5 injection. (c) Quantification of DG volume in PBS-Cy5- and Tau-Cy5-injected mice. (d) Number of apoptotic fractin ${ }^{+}$cells in the DG. (e, $\mathbf{f}$ ) Representative images (e) and quantification ( $\mathbf{f}$ ) of the number of mature granule neurons in PBS-Cy5- and Tau-Cy5-injected mice. Tau-Cy5 injection did not lead to differences in any of these general morphometric parameters. In (d, f) graphs represent mean \pm SEM; $n=6$ mice per experimental condition. DG, dentate gyrus; $\mathrm{GL}$, granular layer; H, hilus. White scale bar: $300 \mu \mathrm{m}$. Red scale bar: $50 \mu \mathrm{m}$. Green scale bar: $10 \mu \mathrm{m}$

\section{Retroviral stock preparation}

We used a retroviral stock encoding for $\mathrm{RFP}^{34}$. The plasmids used to produce the virus were kindly provided by Prof. FH. Gage and Dr. F. Calegari. Retroviral stocks were concentrated to working titers of $1 \times 10^{7}-2 \times 10^{8}$ $\mathrm{pfu} / \mathrm{ml}$ by ultracentrifugation ${ }^{35}$. Since the retroviruses used are engineered to be replication-incompetent, only cells dividing at the time of surgery are infected ${ }^{35}$. In the DG, these proliferative cells are almost exclusively restricted to newborn granule neurons ${ }^{35}$.

\section{Stereotaxic surgery}

Mice were anesthetized with isoflourane and placed in a stereotaxic frame. Retroviruses were injected into the DG at the following coordinates $(\mathrm{mm})$ relative to bregma in the anteroposterior, mediolateral, and dorsoventral axes: $[-2.1, \pm 1.4,2.2]$. Either PBS-Cy5 or Tau-Cy5 was injected into the DG at the following coordinates $(\mathrm{mm})$ relative to bregma in the anteroposterior, mediolateral, and dorsoventral axes: $[-1.6, \pm 1.4,2.2]$. Two microliters of retrovirus, PBS-Cy5 or Tau-Cy5 solution was infused at a rate of $0.2 \mu \mathrm{l} / \mathrm{min}$ via a glass micropipette. To avoid any suction effect of the solution injected, micropipettes were kept in place at the site of injection for an additional $5 \mathrm{~min}$ before being slowly removed. All animals were 8 weeks old at the time of injection.

\section{Administration of the thymidine analog IdU}

The thymidine analog IdU (Sigma-Aldrich) was used to analyze the survival of 12-week-old newborn granule neurons. IdU was administered over $24 \mathrm{~h}$ diluted in drinking water at $0.92 \mathrm{mg} / \mathrm{ml}$. These doses were based on equimolar doses of $0.8 \mathrm{mg} / \mathrm{ml} \mathrm{BrdU}^{36}$.

\section{Behavioral tests}

- Elevated Plus Maze (EPM) test: animals were tested in a 5-min single trial of the EPM (Cibertec, Madrid, Spain) paradigm, as a measurement of anxiety-like behavior, as previously described ${ }^{37}$. Briefly, mice were allowed to move freely along the apparatus under constant intense white light. Animal movement was video-recorded using Anymaze (Stoelting, USA) software and analyzed automatically by the software. The total time spent in the open arms and the total distance covered are shown in the graphs.

- Open Field (OF) and Novel Location Preference (NLP) tests: animals were placed in a square $(45 \times 45 \mathrm{~cm})$, constantly illuminated, open-field methacrylate arena. The test was performed on three consecutive days, on which animals were subjected to a single 10-min trial. On the first day, they were placed inside the arena and allowed to explore it (habituation) (OF phase). During the second day (sample phase), two identical objects were placed symmetrically in the central part of the arena. On the third day (test phase), one of the objects (newly located object) was moved to a peripheral position, while the other one remained in its original position. Animal performance was video-recorded using Anymaze (Stoelting, USA) software and analyzed automatically by the software. Total distance moved, speed, time immobile, number of immobile episodes, entries to the center and time in the center on day 1 are shown in the graphs. Memory index (percentage of time exploring the novel-located object/ total time exploring novel + unaltered object) on day 3 is shown in the graphs.

\section{Sacrifice}

Mice were fully anesthetized with an intraperitoneal pentobarbital injection (EutaLender, $60 \mathrm{mg} / \mathrm{kg}$ ). Animals belonging to group A were transcardially perfused with $0.9 \%$ saline followed by $4 \%$ paraformaldehyde in phosphate buffer. Brains were removed and post-fixed overnight in the same fixative. Animals belonging to the group B were perfused only with $0.9 \%$ saline. Brains were removed, and left and right hemispheres were separated. Left hemispheres were used for biochemical analyses. 
Hippocampi were quickly dissected on ice and frozen in dry ice ( $n=9$ animals per experimental group). Right hemispheres were used for histological or electron microscopy analyses. For histological determinations, right hemispheres of six animals belonging to each experimental group were immersed in $4 \%$ paraformaldehyde overnight. For electron microscopy determinations, the right hemisphere of the three remaining animals belonging to each experimental group was immersed in $4 \%$ paraformaldehyde $+2 \%$ glutaraldehyde in phosphate buffer overnight.

\section{Immunohistochemistry}

Sagittal brain sections were obtained on a Leica VT1200S vibratome $(50-\mu m$-thick sections). For immunohistochemical analysis, series of brain slices were randomly made up of one section from every ninth. Slices were initially pre-incubated in phosphate buffer with $1 \%$ Triton X-100 and 1\% bovine serum albumin, and dual immunohistochemistry was then performed as described previously $^{23}$, using the following primary antibodies: rabbit anti-RFP (Millipore, 1:2000); mouse anti-Cy5 (Abcam, 1:500); goat anti-Doublecortin (DCX) (Santa Cruz, 1:500); rabbit anti-Fractin (a fragment of actin cleaved by caspase 3) (BD Biosciences, 1:500); mouse antiBrdU/IdU (BD Biosciences, 1:500); rabbit anti-MAP-2 (Synaptic Systems, 1:500); and rabbit anti-NeuN (Millipore, 1:1000). To detect the binding of primary antibodies, Alexa-488 donkey anti-mouse and Alexa-555 donkey anti-rabbit (Invitrogen, 1:1000) secondary antibodies were used. All the sections were counterstained for $10 \mathrm{~min}$ with DAPI (Merck, 1:5000) in order to label nuclei.

\section{Electron microscopy}

After a fixation step, 200- $\mu \mathrm{m}$ sagittal sections were obtained on a Leica VT1200S vibratome. Four sections per mouse containing the whole hippocampus were postfixed in $2 \%$ osmium tetroxide $\left(\mathrm{OsO}_{4}\right)$ for $2 \mathrm{~h}$. They were then rinsed, dehydrated, and embedded in Durcupan (Durcupan; Fluka). Serial semi-thin sections $(1 \mu \mathrm{m})$ were cut with a diamond knife and stained with $1 \%$ toluidine blue. Subsequently, the area of interest was trimmed, and ultrathin sections $(0.06 \mu \mathrm{m})$ were obtained with a diamond knife. These sections were then stained with lead citrate and examined under a JEM1010 Jeol electron microscope equipped with a $4 \mathrm{Kx} 4 \mathrm{~K}$ TemCam-F416 Digital camera. All the images were obtained at $\times 15,000$ magnification. In order to analyze the afferent synapses of the hippocampal granule neurons, 30 images per animal containing the molecular layer $(\mathrm{ML})$ were obtained. With respect to the ultrastructural organization of the efferent synapses of these neurons, at least 30 images per animal containing the stratum lucidum and the inner boundary of the stratum pyramidale of the CA3 region were obtained.

The density of synapses (number of synapses per $\mu \mathrm{m}^{2}$ ) and the area of the postsynaptic density (PSD) were measured in each sub-region separately. In addition, the density of presynaptic vesicles in the presynaptic terminal (number of vesicles per $\mu \mathrm{m}^{2}$ ) and the number of vesicles fused with the active zone were measured. Given that some presynaptic terminals were not fully included in the image due to the high complexity and size of these structures, a circular region of interest was used to count the number of vesicles and to calculate their density. The different parameters were measured manually in the images using ImageJ software. The density of synapses was calculated by dividing the total number of synapses in each image by the known area of the image. 100-150 synapses per experimental group and region were analyzed.

\section{Volume estimation of the dentate gyrus}

To measure the DG volume, we used a semi-automatic Cavalieri system (ImageJ v.1.47, NIH, USA, http://rsbweb. nih.gov/ij/) in a series of $50-\mu \mathrm{m}$ sections stained with DAPI, as previously described.

\section{Cell counts}

The total number of mature granule neurons was calculated under a LSM710 Zeiss confocal microscope $(\times 63$ oil immersion objective) using the physical dissector method adapted for confocal microscopy ${ }^{23}$. Briefly, five stacks of images per animal were used to determine the density of mature granule neurons in a reference structure of known volume $(30(X) \times 30(Y) \times 10(Z) \mu \mathrm{m})$. The average density of these cells was then multiplied by the total volume of the DG in order to calculate the total number of mature granule neurons per animal ${ }^{38}$.

The total number of $\mathrm{DCX}^{+}$neuroblasts was calculated using the physical dissector method adapted for confocal microscopy (Zeiss LSM710), as previously described ${ }^{38}$. Six stacks of images randomly selected from one series of slices were examined per animal.

The total number of $\mathrm{IdU}^{+}$and fractin ${ }^{+}$cells was counted under an optical fluorescence microscope (Zeiss Axioskop2) using the optical-dissector method, as previously described ${ }^{39}$.

In order to study the maturation of newborn neurons, the percentage of 8-week-old $\mathrm{RFP}^{+}$newborn neurons that expressed NeuN was analyzed in $\times 60$ magnification $(2 \times$ zoom) confocal images. A minimum of 100 cells per experimental condition were examined.

\section{Colocalization between Cy5 signal and MAP-2}

Colocalization between Cy 5 and MAP-2 was measured in the DG of stereotaxically injected mice. For this 
purpose, 16-bit confocal images of the DG $(\times 60$ oil objective, $2 \times$ zoom) were obtained under a Zeiss LSM710 confocal microscope. Five images per animal were acquired. Images were analyzed by means of the $\mathrm{JaCoP}$ colocalization plugin for Image ${ }^{40}$. Briefly, images were subjected to an invariant threshold. The plugin analyzed the colocalization area above the threshold between the $\mathrm{Cy} 5^{+}$or $\mathrm{MAP}-2^{+}$, and Mander's coefficients were calculated and shown in the graphs.

\section{Morphometric analysis of newborn granule neurons}

For immunohistochemical analysis, series of $50-\mu \mathrm{m}$ brain sections were made up randomly of one section from every ninth. Three series of sections from each animal were used for the immunohistochemical detection of RFP. Fifty randomly selected neurons from each experimental condition were reconstructed under a LSM710 Zeiss confocal microscope $(\times 25$ oil immersion objective). Confocal stacks of images were obtained ( $Z$ axis interval: $2 \mu \mathrm{m}$ ), and Z-projections were analyzed in order to determine total dendritic length and degree of dendritic arbor branching (Sholl's analysis). All cells were traced using NeuronJ plugin for ImageJ software. Sholl's analysis was performed using the plugin ShollAnalysis for ImageJ. This analysis consists of placing a central point on the cell soma and tracing concentric circles (separated by a distance interval of $10 \mu \mathrm{m}$ ). The number of times that the dendritic tree intersected each circle is shown in the graphs (number of crossings). The percentage of cells aberrantly showing several primary apical dendrites was calculated as previously described ${ }^{23}$. In addition, in order to measure the migration of retrovirus-labeled newborn granule neurons, the distance between the hilar border of the subgranular zone and the center of the cell nucleus of each $\mathrm{RFP}^{+}$cell was measured as previously described ${ }^{41}$. One hundred cells per genotype were analyzed.

\section{Morphometric analysis of the dendritic spines of newborn granule neurons}

Dendritic spines were analyzed in 8-week-old retrovirus-labeled newborn neurons. Confocal stacks of images were obtained in a LSM710 Zeiss confocal microscope ( $\times 63$ oil immersion objective; $X Y$ dimensions: $67.4 \mu \mathrm{m} ; Z$-axis interval: $0.2 \mu \mathrm{m})$. The dendritic length of each segment was measured on Z-projections, and the number of dendritic spines was counted using the NeuronStudio software (CNIC, Mount Sinai School of Medicine, 2007-2009) ${ }^{42}$. Prior to spine analysis, images were deconvoluted using the Huygens Professional software (Scientific Volume Imaging). A minimum of 100 dendrites per experimental group were examined. Dendritic fragments were automatically constructed using NeuronStudio software, and then individual seed points were rectified manually to more accurately trace the dendrite.
Thereafter, the dendritic spines were detected by the software and assigned to one of the following three categories: stubby, thin, and mushroom, as previously described $^{3,41}$. Each spine was checked manually in order to assure accurate classification. The total density of spines (number of spines/ $\mu \mathrm{m}$ ), the number and percentage of each type of spine, the spine head diameter, and the approximate spine length (Max-DTS) were calculated for each type of spine, as previously described ${ }^{41}$.

\section{Area of mossy fiber terminals of newborn granule neuronsThe abbreviation MFT has been deleted from the heading and introduced in the text, please confirm.OK}

The area of individual mossy fiber terminals (MFTs) was measured in the CA3 region of RFP-injected animals. A minimum of 20 stacks of images per experimental condition were obtained in a LSM710 Zeiss confocal microscope $(\times 63$ oil immersion objective; $X Y$ dimensions: $100 \mu \mathrm{m}$; Z-interval: $0.5 \mu \mathrm{m})$. Stacks were randomly obtained from the sections comprising the series. Zprojections were obtained, and the area of each MFT was measured manually using ImageJ software, as previously described $^{3,43}$. A minimum of 100 MFTs per experimental condition were measured.

\section{Statistical analysis}

Statistical analysis was performed using the SPSS 23 software (SPSS, 1989; Apache Software Foundation, Chicago, IL, USA). The Kolmogorov-Smirnov test was used to test the normality of the sample distribution. Data were analyzed by a Student's $t$-test in the case of normal sample distribution, or by a nonparametric test (Mann-Whitney $U$ test) in those cases in which normality could not be assumed. Graphs represent mean values \pm SEM. The analysis of the percentage of the types of spine was accomplished by a chi-squared $\left(\chi^{2}\right)$ test.

\section{Results}

The presence of Tau-Cy5 does not cause significant alterations in gross anatomy of the DG

A schematic representative diagram of the experimental design is shown in Fig. 1a. Briefly, animals were subjected to their respective stereotaxic surgeries and 8 weeks later sacrificed. Figure $1 \mathrm{~b}$ shows representative tile-scan images of the whole hippocampal region of animals injected with either PBS-Cy5 or Tau-Cy5. Tau-Cy5-injected animals showed an intense Cy5 signal at this time point, whereas this signal was mostly absent in mice injected with PBSCy5. In order to confirm the soluble nature of Tau, an analytic ultracentrifugation assay was performed. This analysis showed that injected Tau was composed mostly of monomers (54\%) and dimers (31.4\%) (Supplementary Figure S1). The presence of intracellular soluble Tau in granule neurons was studied by measuring the 
colocalization between Cy5 signal and MAP-2 staining in the DG. As shown in Supplementary Figure S2A,B, an increased signal of Cy5 colocalizing with MAP-2 staining was observed in Tau-Cy5-injected animals in comparison to PBS-Cy5-injected ones $(U=1000 ; p=0.009)$. These observations thus reveal the presence of intracellular Tau in the somatodendritic compartment of these cells. Mander's coefficients are shown in Supplementary Figure S2B. To rule out the possibility that the stereotaxic injection of soluble Tau triggers massive neurodegeneration of the region, we measured the volume of the DG in both animal groups. No changes in this parameter were observed ( $t=0.332 ; p=0.745$ ) (Fig. 1c). In order to rule out the possibility that soluble Tau injections trigger the generalized apoptosis of granule neurons, the number of apoptotic fractin $^{+}$cells was calculated in Tau-Cy5- and PBS-Cy5-injected animals $(U=5.000 ; p=0.486)$ (Fig. 1d). In addition, the total number of mature granule neurons remained unaltered after Tau-Cy5 injection $(t=0.702 ; p$ $=0.493$ ) (Figs. 1e, f).

To further characterize the effects of soluble Tau injection on the general rate of AHN, we counted the number of $\mathrm{DCX}^{+}$neuroblasts, finding no significant changes in this parameter $(t=0.524 ; p=0.609)$ (Supplementary Figure $3 \mathrm{~A}, \mathrm{~B})$. Moreover, we studied the longterm survival of newborn granule neurons by quantifying the number of 12 -week-old $\mathrm{IdU}^{+}$cells in the DG (Supplementary Figure 3C). No changes in this parameter were found $(U=4.000 ; p=0.629)$. Given the absence of changes in the aforementioned parameters, we conclude that the presence of soluble Tau-Cy5 does not cause dramatic alterations in the gross anatomy of the DG or in the general rate of AHN.

\section{Tau-Cy5 impairs the connectivity of granule neurons at the ultrastructural level}

To study whether the stereotaxic injection of soluble Tau in the DG causes long-term alterations in the synapses of granule neurons, we analyzed the afferent (Figs. 2a-e) and efferent (Figs. 2f-j) synapses of these cells at the ultrastructural level in the ML of the DG and in the CA3 hippocampal regions, respectively. Figure 2a shows representative electron microscopy images of the ML of animals injected with either PBS-Cy5 or Tau-Cy5. In this region, stereotaxic injection of soluble Tau caused a reduction in the density of synapses (Fig. 2b) $(U=3998.5$; $p=0.044)$. Moreover, the area of the PSDs of the remaining synapses increased (Fig. 2c) $(U=2399 ; p<$ $0.001)$, whereas both the number of synaptic vesicles fused to the active zone (Fig. $2 \mathrm{~d})(t=6.282 ; p<0.001)$ and the density of vesicles in the presynaptic terminal (Fig. 2e) $(U=170 ; p=0.028)$ decreased in animals injected with Tau-Cy5. Figure $2 \mathrm{f}$ shows representative images of the CA3 region of animals injected with either PBS-Cy5 or
Tau-Cy5. Stereotaxic injection of soluble Tau did not alter the density of synapses (Fig. $2 \mathrm{~g})(U=2285.5 ; p=0.419)$ but did lead to an increase in the area of the PSDs (Fig. 2h) $(U=1230 ; p<0.001)$ and to a marked reduction in both the number of synaptic vesicles fused to the presynaptic active zone (Fig. $2 \mathrm{i})(U=337 ; p=p<0.001)$ and the density of synaptic vesicles in the presynaptic terminal (Fig. 2 j) $(t=4.553 ; p<0.001)$. In summary, stereotaxic injection of soluble Tau triggered alterations in the number and ultrastructure of synapses of the general population of granule neurons.

\section{Tau-Cy5 alters the morphology of newborn granule neurons}

To study whether soluble Tau has an additional impact on the morphology of newly generated granule neurons, we injected an RFP-expressing retrovirus into the DG of animals injected with PBS-Cy5 or Tau-Cy5 (Fig. 1a). After the complete maturation of these cells (8 weeks postinjection), the animals were sacrificed. To ensure that the cells analyzed were at the same developmental stage in PBS-Cy5- and Tau-Cy5-injected animals, the percentage of $\mathrm{RFP}^{+}$neurons that expressed the mature neuron marker NeuN was calculated. The vast majority of cells were $\mathrm{NeuN}^{+}$in both experimental groups (this percentage was $94 \%$ in the case of PBS-Cy5-injected animals and 97\% in the case of Tau-Cy5-injected mice) (Supplementary Figure S3C). These observations thus indicate that the cells analyzed were at the same developmental stage. Morphometric determinations were then performed in RFP-labeled newborn granule neurons. Figure 3a shows representative images of the morphology of newborn granule neurons of the two groups of animals. Stereotaxic injection of soluble Tau caused a reduction in the total dendritic length of newborn granule neurons (Fig. 3b) $(t$ $=4532 ; p \leq 0.001$ ) and altered the morphology of the whole dendritic tree, as revealed by Sholl's analysis (Fig. 3c). Statistical comparisons of Sholl's analysis corresponding to each $10-\mu \mathrm{m}$ length dendritic segment are shown in Supplementary Table 1. According to these morphological alterations, the percentage of newborn granule neurons showing more than one primary apical dendrite increased (Fig. 3d) $\left(\chi^{2}=17.828 ; p \leq 0.001\right)$ and the length of the primary apical dendrite decreased (Fig. 3e) $(U=1763 ; p \leq 0.001)$ in Tau-Cy5-injected animals. In addition, the migration of these neurons into the GL increased (Fig. 3f) $(U=2522 ; p=0.006)$.

\section{Tau-Cy5 impairs the connectivity of newborn granule neurons}

To address whether the stereotaxic injection of soluble Tau causes long-term effects on the connectivity of adultborn granule neurons, we studied the number, size and subtypes of dendritic spines of RFP-labeled newborn 


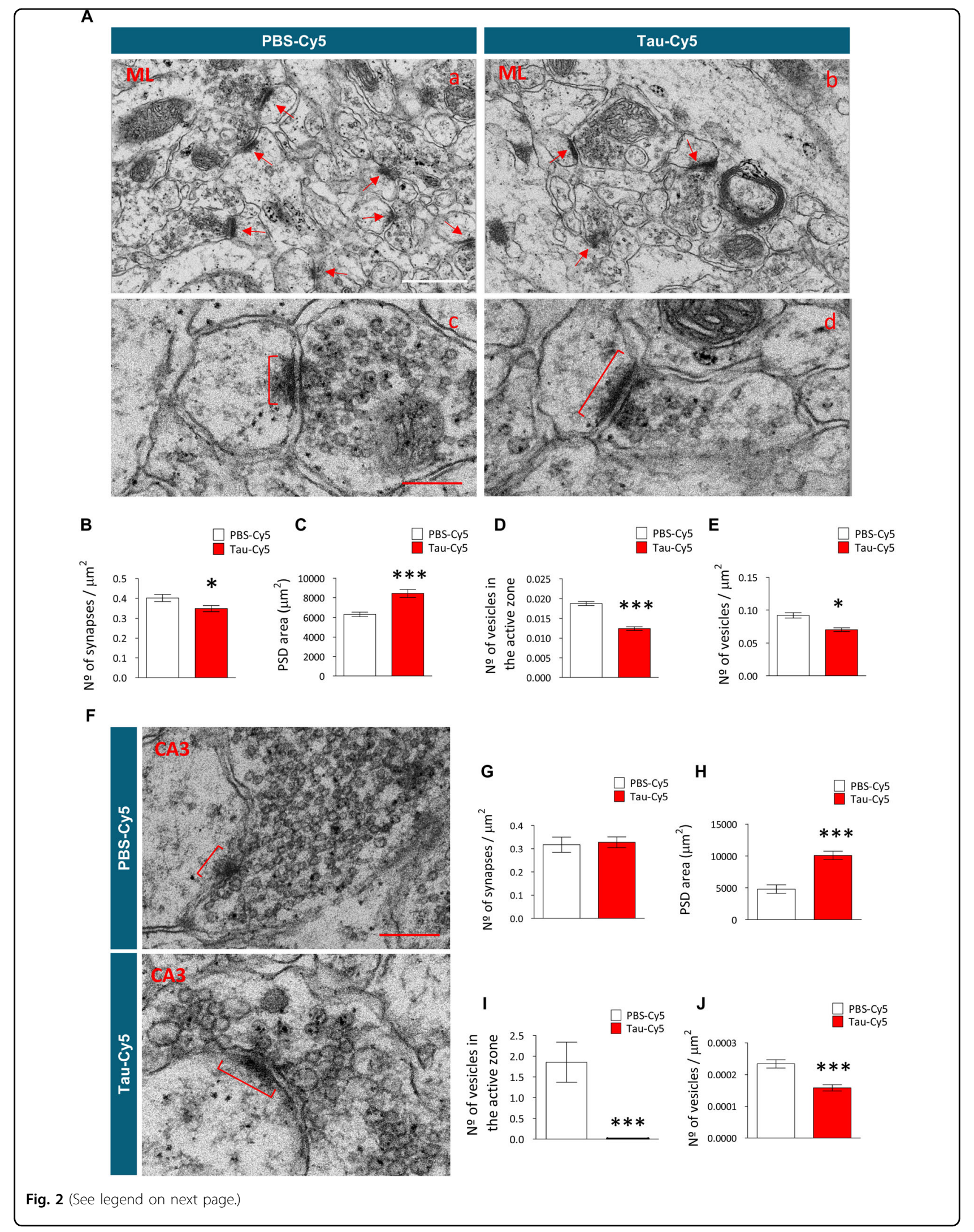




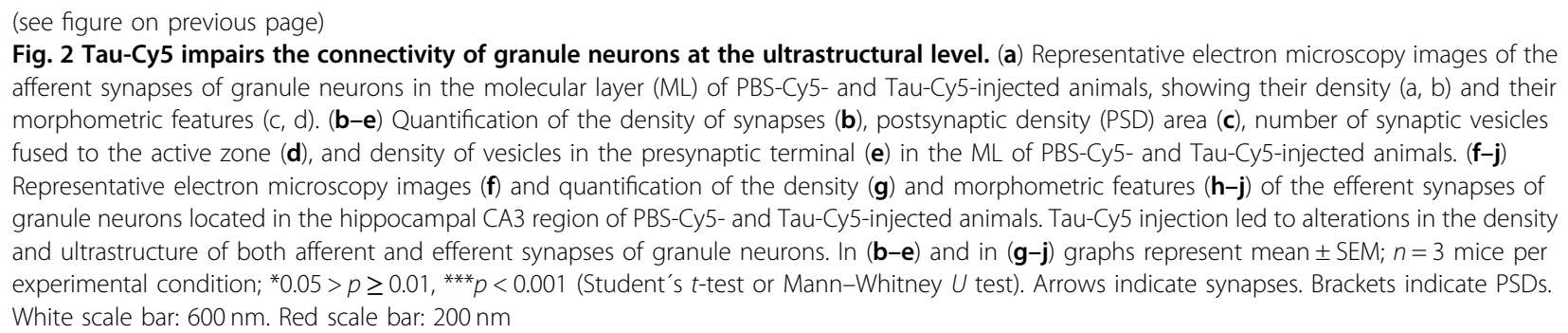

granule neurons of animals injected with PBS-Cy5 or Tau-Cy5 (Fig. 4). Figure 4a shows representative images of dendritic fragments of newborn granule neurons of animals injected with PBS-Cy5 or Tau-Cy5. Stereotaxic injection of soluble Tau reduced the density of dendritic spines (Fig. 4b) $(U=8153 ; p \leq 0.001)$. In addition, the remaining spines showed an increase in head volume (Fig. 4c) $(U=10472778 ; p \leq 0.001)$, whereas the approximate measurement of spine length (Max-DTS) decreased (Fig. 4d) $(U=10619616.5 ; p \leq 0.001)$. According to morphological criteria, dendritic spines are classified into stubby, thin and mushroom subtypes ${ }^{41}$. Figure 4 e shows that the stereotaxic injection of soluble Tau caused a marked increase in the percentage of stubby spines $\left(\chi^{2}=\right.$ 73.036; $p$ 5.001), whereas the percentage of mushroom spines was drastically reduced $\left(\chi^{2}=52.761 ; p \leq 0.001\right)$. Next, we studied various morphometric parameters of each spine type separately (Figs. 4f-k). Tau-Cy5 increased the head volume of each spine type (stubby (Fig. 4f) $(U=$ 204,728; $p \leq 0.001)$, thin (Fig. 4g) $(U=139,626 ; p \leq 0.001)$ and mushroom (Fig. 4h) $(U=376,153 ; p \leq 0.001))$ and reduced the Max-DTS of thin (Fig. 4j) $(U=75,118 ; p \leq$ $0.001)$ and mushroom (Fig. 4k) $(U=256,459 ; p \leq 0.001)$ spines. Finally, the area of the axonal terminals (mossy fiber terminals, MFTs) of newborn granule neurons was measured in the CA3 hippocampal subfield of PBS-Cy5and Tau-Cy5-injected animals. Tau-Cy5 injection reduced the area of MFTs $(U=7700.5 ; p=0.03)$ (Figs. 4l, $\mathrm{m})$.

\section{Tau-Cy5 impairs behavioral pattern separation}

Newborn granule neurons play a relevant role in hippocampal-dependent memory ${ }^{18,44,45}$, and they are particularly important for behavioral pattern separation skills $^{24}$. Given the alterations observed in the connectivity and the morphology of these cells, we questioned whether behavioral pattern separation capacity was also impaired in the mice. The novel location preference (NLP) test is considered a reliable model of pattern separation in rodents $^{37,46,47}$. The stereotaxic injection of Tau-Cy5 reduced the percentage of time spent exploring the object placed in a new location (Memory index, $t=3.364$; $p=0.007)$, thus indicating impaired behavioral pattern separation ability in these mice (Fig. 5a). However, we questioned whether these alterations could be due to either anxiety-like behavior or motor impairment of these animals. To explore these possibilities, we performed Elevated Plus Maze (EPM) and Open Field (OF) tests respectively. Soluble Tau-Cy5 did not alter anxiety-like behavior in the EPM test, as Tau-Cy5-injected animals displayed a percentage of time in open arms comparable to that of their PBS-Cy5-injected counterparts ( $t=$ $-0.339 ; p=0.739$ ) (Fig. $5 \mathrm{~b}$ ). In addition, the behavior of the mice in the OF test remained unaltered after the stereotaxic injection of Tau-Cy5. Soluble Tau did not change parameters related to motor behavior, such as the total distance traveled (Fig. 5c) $(t=-0.091 ; p=0.929)$, mean speed (Fig. 5d) $(t=-0.076 ; p=0.940)$, time immobile (Fig. 5e) $(t=-0.595 ; p=0.561)$ and number of immobile episodes (Fig. 5f) $(t=1.370 ; p=0.195)$. In addition, it did not alter those related to anxiety-like behavior, such as the number of entries to the center (Fig. 5g) $(t=0.545 ; p=0.593)$ or the time spent in the center of the arena (Fig. 5h) $(t=0.765 ; p=0.458)$. On the basis of these observations, we can conclude that the impairment in the behavioral pattern separation skills of the mice injected with Tau-Cy5 was specific and not due to a non-selective alteration in their general behavior.

\section{Discussion}

Tau is a neuronal MAP that plays numerous physiological roles ${ }^{48}$. In recent decades Tau functions were believed to be limited to the axonal compartment ${ }^{49-54}$. However, growing evidence supports the relevance of Tau also in the neuronal somatodendritic compartment ${ }^{3,5,55}$. In this regard, we have recently shown that Tau is required for in vivo afferent synapse formation in newborn granule neurons ${ }^{3}$. Although the absence of Tau has been reported to confer neuroprotection, we have also shown that this MAP is required for the stimulatory effects of certain external stimuli on $\mathrm{AHN}^{3}$. Thus, the current belief in the field is that a tight regulation of Tau 

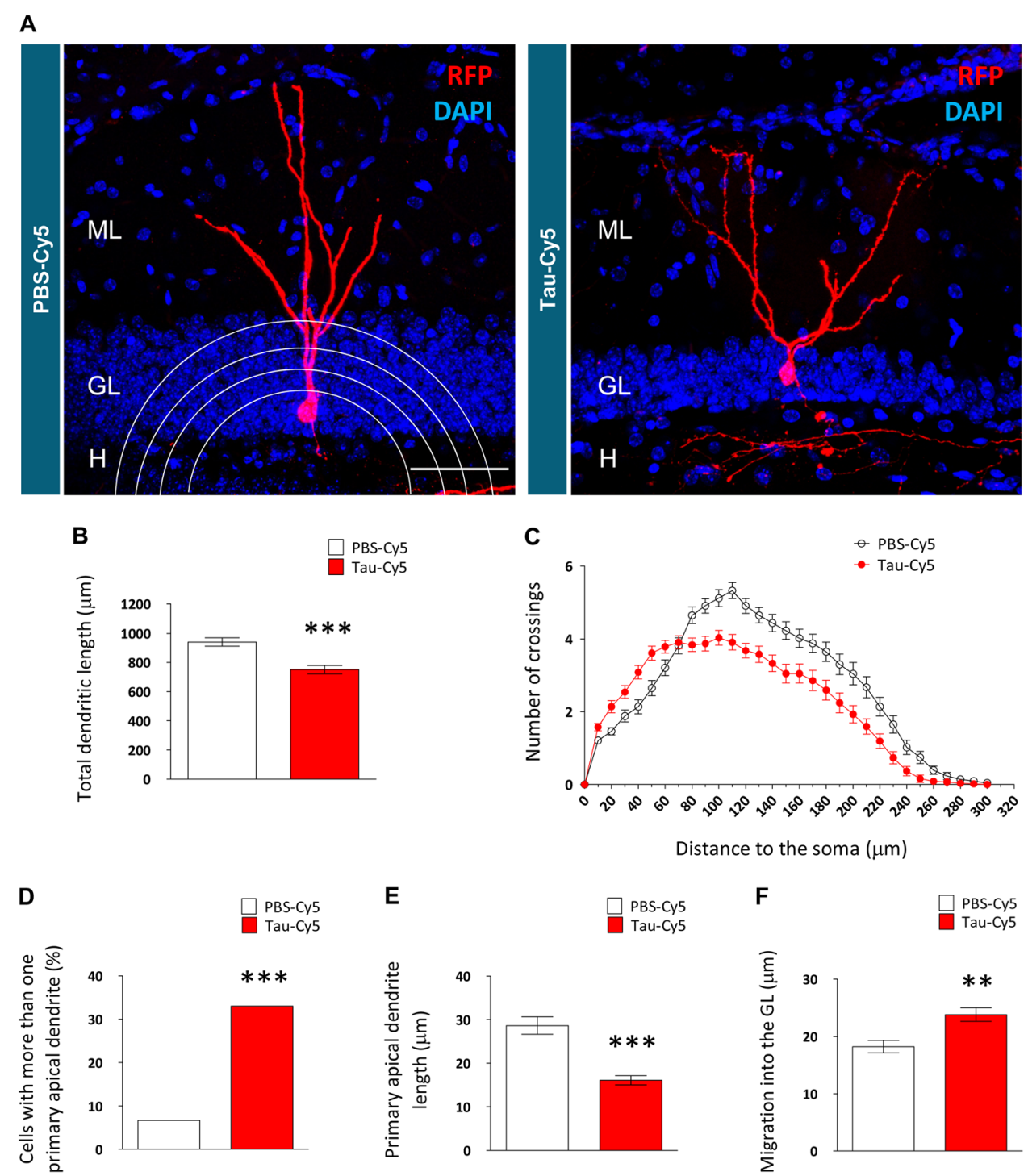

Fig. 3 The presence of Tau-Cy5 alters the morphology of newborn granule neurons. (a) Representative images of RFP-labeled 8-week-old newborn granule neurons in PBS-Cy5- and Tau-Cy5-injected mice. A schematic representation of Sholl's analysis is shown in the image corresponding to a PBS-Cy5-injected mouse. (b-f) Quantification of total dendritic length (b), Sholl's analysis (c), percentage of neurons with several primary apical dendrites (d), length of the apical dendrite (e), and migration into the GL (f) of newborn granule neurons in PBS-Cy5- and Tau-Cy5injected mice. Tau-Cy5 injection altered the morphology and migration of newborn granule neurons in the DG. ML, molecular; $G L$, granular layer; $H$, hilus. In (b, $\mathbf{c}$, e and $\mathbf{f}$ ) graphs represent mean $\pm \mathrm{SEM}$; ${ }^{* *} 0.01>p \geq 0.001,{ }^{* * *} p<0.001$ (Student's $t$-test or Mann-Whitney $U$ test). Graph in (d) represent percentage; ${ }^{* *} p<0.001$ ( $x^{2}$ test). $N=4$ mice per experimental condition. Scale bar: $50 \mu \mathrm{m}$

metabolism is required for each neuron to achieve the appropriate balance between stability and plasticity needed at any given time. In line with the aforementioned hypothesis, post-transcriptional changes occurring in Tau protein have drastic consequences on its physiological functions. For instance, Tau phosphorylation reduces its affinity to bind microtubules, which leads to microtubule destabilization and cytoskeleton instability ${ }^{56-61}$. Although this instability may occur in neurons during discrete periods of time in which a higher degree of plasticity is required, chronic dysregulation of Tau phosphorylation has severe effects on neuronal function ${ }^{57,62,63}$. An important point to bear in mind is the link between Tau phosphorylation and the capacity of this protein to form insoluble aggregates ${ }^{10,64}$. On the one hand, phosphorylation can induce Tau aggregation in vitro, probably due to the addition of negative charges ${ }^{65,66}$. Moreover, it is also believed that Tau detachment from microtubules can also trigger the aggregation of this protein by allowing greater interaction between phosphorylated and non- 


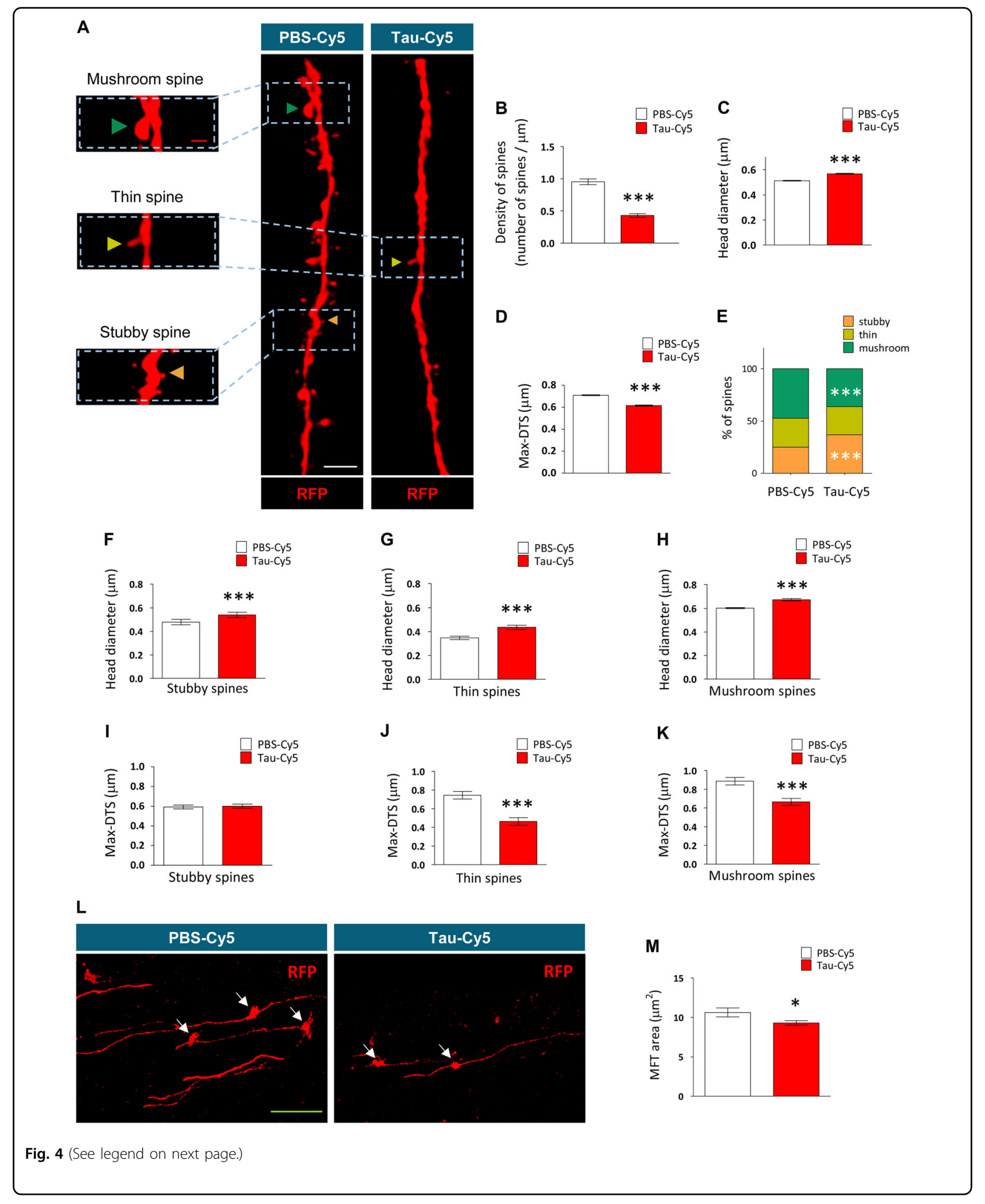


(see figure on previous page)

Fig. 4 Tau-Cy5 impairs the connectivity of newborn granule neurons. (a) Representative images of 8-week-old RFP-labeled newborn granule neuron dendrites of PBS-Cy5- and Tau-Cy5-injected mice showing their dendritic spines. The distinct spine morphologies-stubby (orange triangles), thin (yellow triangles), and mushroom (green triangles) -are shown in high-power magnifications. (b-d) Quantification of the density of dendritic spines (b), spine head volume (c) and spine Max-DTS (d) of newborn granule neurons of PBS-Cy5- and Tau-Cy5-injected mice. Tau-Cy5 injection decreases the density of dendritic spines and alters their morphology. (e) Quantification of the percentages of stubby, thin and mushroom spines in newborn granule neurons of PBS-Cy5- and Tau-Cy5-injected mice. Tau-Cy5 injection altered the proportion of the different types of spines, decreasing that of mushroom spines and increasing that of stubby ones. (f-h) Quantification of the head diameter of stubby (f), thin ( $(\mathbf{g})$ and mushroom (h) dendritic spines of newborn granule neurons of PBS-Cy5- and Tau-Cy5-injected mice. (i-k) Quantification of the Max-DTS of stubby (i), thin $\mathbf{( j )}$ and mushroom (k) dendritic spines of newborn granule neurons of PBS-Cy5- and Tau-Cy5-injected mice. Tau-Cy5 injection increased the head diameter of every type of spine and decreased the Max-DTS of thin and mushroom ones. (I, $\mathbf{m})$ Representative images (I) and quantification ( $\mathbf{m})$ of the newborn neuron MFT area in the CA3 region of PBS-Cy5- and Tau-Cy5-injected mice. Tau-Cy5 injection reduced the area of newborn neuron MFTs. Graphs in (b-d, f-k, m) represent mean \pm SEM; ${ }^{*} 0.05>p \geq 0.01,{ }^{* * *} p<0.001$ (Mann-Whitney $U$ test). Graph in (e) represent percentages. ${ }^{* * *} p<0.001$ ( $x 2$ test). $n=4$ mice per experimental condition. White scale bar: $3 \mu \mathrm{m}$. Red scale bar: $1 \mu \mathrm{m}$. Green scale bar: $20 \mu \mathrm{m}$

phosphorylated Tau monomers ${ }^{65,66}$. Importantly, growing evidence suggests that Tau oligomers are also highly toxic for neurons, and a prion-like mechanism has been proposed to explain the spread of Tau from neuron to neuron during neurodegenerative processes ${ }^{11}$. In the present work, we aimed to address the effects of non-aggregated, soluble Tau on the structural plasticity of the granule neurons of the DG. Despite the soluble nature of the TauCy5 used in this study ${ }^{67}$, we have demonstrated a substantial in vivo detrimental effect caused by the exposure of granule neurons to this soluble protein. These neurons showed markedly decreased connectivity (reduced number of synapses) in the ML. It should be noted that the synaptic contacts with the perforant pathway occurring in the $\mathrm{ML}$ are the main afferent connections received by granule neurons from the Entorhinal cortex. Hence, these contacts are critical for the synaptic integration of granule neurons in the trisynaptic circuit ${ }^{68-72}$. Our data show that the phenomenon of reduced afferent connectivity also occurs in newborn granule neurons, which exhibited a reduced number of dendritic spines. Interestingly, this reduction is attributable mainly to the specific reduction of mushroom spines, as the number of thin spines remained unchanged while stubby spines increased in number. Of note, mushroom spines are considered to harbor the strongest synapses in the central nervous system, whereas thin and stubby spines are thought to be more plastic and to harbor weaker or more immature synapses $^{3,73}$. Hence, the marked decrease in the number of mushroom spines, together with the increase in the proportion of stubby spines, may lead to decreased synaptic efficiency and instability of the global network at this level. Interestingly, at the ultrastructural level, synapses in the ML displayed a higher PSD area, which is in agreement with the increase observed in the head volume of dendritic spines of newborn granule neurons. However, given the marked depletion of synaptic vesicles at the presynaptic level and the reduced number of synapses, uncoupling between the presynaptic and the postsynaptic elements may occur in the ML of Tau-Cy5injected animals. Alternatively, the increased PSD area and spine head volume could be interpreted as a compensatory mechanism through which to counteract the reduced synaptic transmission caused by soluble Tau. However, despite this putative attempt to compensate for reduced synaptic efficiency, the final outcome of the scenario is a clear impairment in behavioral pattern separation ability. In agreement with the proposed decrease in synaptic transmission, it should be mentioned here that the area of the MFTs was reduced by soluble Tau, thus supporting the notion that newborn granule neurons provide an insufficient contribution to the hippocampal trisynaptic circuit.

In line with this, it has been proposed that newly born neurons contribute to the creation of memory representations in the hippocampal network by enhancing pattern separation ${ }^{24,26}$. Although the specific contribution of both developmentally generated and newly born granule neurons to the behavioral impairment cannot be evaluated separately in the present paradigm, it can be hypothesized that the alterations in structural plasticity in Tau-Cy5-injected animals are, to some extent, responsible for the impaired capacity of these animals to perform behavioral pattern separation.

In addition, another relevant aspect believed to be critical for newborn granule neuron functionality is their morphology $\mathrm{y}^{74}$. In this regard, certain pathological conditions trigger the appearance of a particular morphological phenotype in newborn granule neurons (namely, the "Vshape" phenotype $)^{23,74}$. Whereas the normal phenotype of these neurons (the "Y-shape") is characterized by the presence of a single primary apical dendrite extensively branched in the ML, "V-shape" newborn granule neurons display several primary apical dendrites emerging from the soma and a markedly reduced distal branching of the dendritic tree ${ }^{23}$. Interestingly, although the causal 

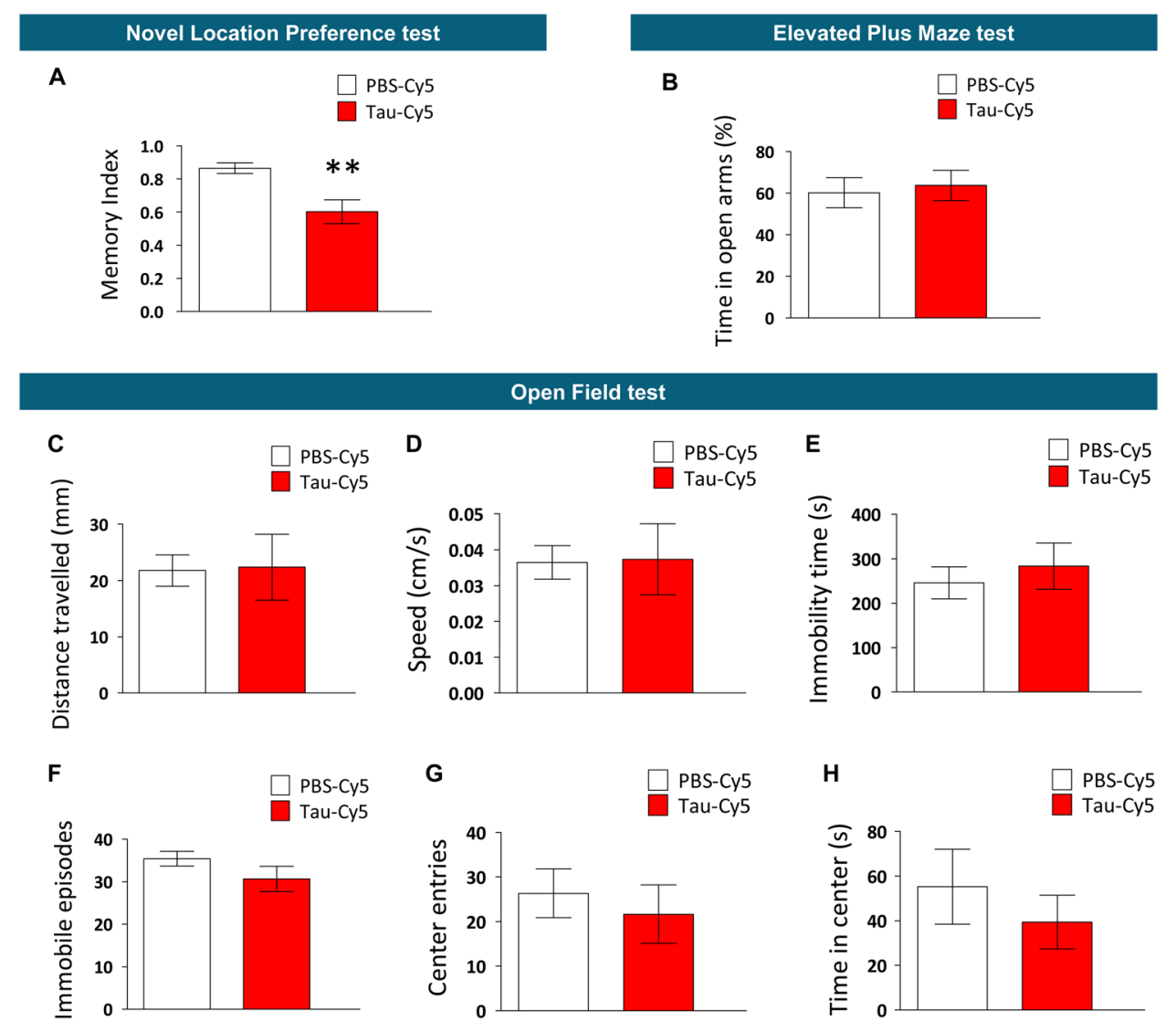

Fig. 5 Tau-Cy5 selectively alters behavioral pattern separation ability but does not cause alterations in the general behavior of the animals. PBS-Cy5- and Tau-Cy5-injected mice were subjected to pattern separation, motor skills and anxiety-like-related behavioral tests. (a) Memory index (time spent exploring the object placed in the new location/total time of exploration) in the Novel Location Preference test. Tau-Cy5 injection impaired pattern separation skills of the mice. (b) Percentage of time spent in the open arms of the Elevated Plus Maze test. Tau-Cy5 injection did not affect anxiety-like behavior. (c-h) Total distance traveled (c), speed (d), immobility time (e), number of immobile episodes (f), and number of entries $(\mathbf{g})$ and time (h) in the center of the arena in the Open Field test. Tau-Cy5 injection did not alter the anxiety-like and motor behavior of the mice. Graphs represent mean $\pm \mathrm{SEM} ; n=9$ mice per experimental condition; ${ }^{* *} 0.01>p \geq 0.001$ (Student's $t$-test)

relationship of these morphological alterations and behavioral impairments has not been established to date, they occur in parallel in diverse animal models of various pathologies ${ }^{74}$. Importantly, the aforementioned "V-shape" phenotype of granule neurons has been observed in the brains of AD patients ${ }^{23}$. Here we show that these morphological features appear in newborn granule neurons exposed to soluble Tau. Whether or not these morphological alterations also contribute to the impairment of behavioral pattern separation skills is an open question that requires further attention.

Noteworthy, increased levels of intracellular Tau are observed in the brains of AD patients ${ }^{75}$. This increase in intracellular Tau is toxic for neurons while a reduction is neuroprotective in animal models of neurodegenerative disorders ${ }^{76}$. Various mechanisms triggered by soluble Tau could be responsible for the alterations in the plasticity of granule neurons observed in the present study ${ }^{77,78}$. On the one hand, microtubule destabilization may have a direct impact on granule neuron morphology, 3,79 , but it may also contribute to the impairment of axonal and dendritic transport of mitochondria and other cargoes to synaptic sites, ${ }^{4,77,78}$. In this regard, we have previously demonstrated that Tau plays a crucial role in regulating mitochondrial axonal transport in hippocampal neurons ${ }^{4}$. This deficient delivery of mitochondria may have important consequences for granule neuron connectivity ${ }^{80}$. In addition, despite the soluble nature of the extracellular Tau injected, a remote possibility of intracellular aggregation cannot be completely ruled out. In this regard, it is also known that excess intracellular Tau activates the unfolded protein response by impairing endoplasmicreticulum-associated degradation of several proteins, including Tau itself ${ }^{81}$. Although Tau has been described to enter nerve cells via several mechanisms ${ }^{67,82,83}$, our group has shown that a principal mechanism of Tau entry 
into neurons includes the stimulation of type M1 and M3 muscarinic acetylcholine receptors ${ }^{84-87}$. Noteworthy, these are the most abundant muscarinic cholinergic receptors expressed in the hippocampus. Tau interaction with these receptors (which are intracellularly coupled to G-proteins belonging to the $G_{q} / G_{11}$ family) triggers calcium entry into the neuron, and, consequently, neuron depolarization $^{88}$. Excessive depolarization is toxic for neurons and may contribute to the additional release of Tau to the extracellular space-a mechanism that has been proposed to exacerbate damage through neuron-toneuron spread throughout the brain.

Nevertheless, here we demonstrate, for the first time, that stereotaxic injection of soluble Tau has detrimental long-term effects on the structural plasticity and connectivity of hippocampal granule neurons. Furthermore, we show that these alterations occur in parallel to a considerable impairment of behavioral pattern separation ability.

\section{Acknowledgements}

The authors would like to thank Esther García and Raquel Cuadros for their technical assistance; Milagros Guerra for her help with electron microscopy sample preparation; the CBMSO animal, electron and confocal microscopy facilities for their help with diverse experimental protocols; and Prof. F.H. Gage and Dr. F. Calegari for kindly providing the retroviral plasmids used in these experiments. The ultracentrifugation assay was performed at the Laboratorio de Ultracentrifugación analítica e interacciones macromoleculares (LUAIM), Universidad Complutense de Madrid, Madrid (Spain).

\section{Funding}

This study was supported by the Spanish Ministry of Economy and Competitiveness (SAF-2014-53040-P (to J.A..) and RYC-2015-17189 (to M.LL.-M.)); the Centro de Investigación Biomédica en Red sobre Enfermedades Neurodegenerativas (CIBERNED, Spain) (to J.Á.); the Alzheimer's Association (2015-NIRG-340709 and AARG-17-528125 (to M.L.-M.)); and the Association for Frontotemporal Degeneration (2016 Basic Science Pilot Grant Award (to M.LL.M.)).

\begin{abstract}
Author details
'Department of Molecular Neuropathology, Centro de Biología Molecular "Severo Ochoa", CBMSO, CSICUAM, Madrid, Spain. ${ }^{2}$ Network Center for Biomedical Research on Neurodegenerative Diseases (CIBERNED), Madrid, Spain. ${ }^{3}$ Department of Molecular Biology, Faculty of Sciences, Universidad Autónoma de Madrid, Madrid, Spain
\end{abstract}

\section{Competing Interests}

The authors declare that they have no competing financial interests.

Publisher's note: Springer Nature remains neutral with regard to jurisdictional claims in published maps and institutional affiliations.

\section{Supplementary information}

The online version of this article (doi:10.1038/s41398-017-0013-6) contains supplementary material.

Received: 28 April 2017 Revised: 3 July 2017 Accepted: 30 July 2017 Published online: 08 December 2017

\section{References}

1. Weingarten, M. D., Lockwood, A. H., Hwo, S. Y. \& Kirschner, M. W. A protein factor essential for microtubule assembly. Proceedings of the National Academy of Sciences of the United States of America 72, 1858-1862 (1975).

2. Conde, C. \& Caceres, A. Microtubule assembly, organization and dynamics in axons and dendrites. Nat. Rev. Neurosci. 10, 319-332 (2009).

3. Pallas-Bazarra, N. et al. Novel function of Tau in regulating the effects of external stimuli on adult hippocampal neurogenesis. EMBO. J. 35, 1417-1436 (2016).

4. Llorens-Martin, M., Lopez-Domenech, G., Soriano, E. \& Avila, J. GSK3beta is involved in the relief of mitochondria pausing in a Tau-dependent manner. PLOS. ONE. 6, e27686 (2011).

5. Ittner, L. M. et al. Dendritic function of tau mediates amyloid-beta toxicity in Alzheimer's disease mouse models. Cell 142, 387-397 (2010).

6. Kimura, $T$. et al. Microtubule-associated protein tau is essential for long-term depression in the hippocampus. Philos. Trans. R. Soc. Lond. B. Biol. Sci. 369, 20130144 (2014).

7. Goedert, M. \& Jakes, R. Expression of separate isoforms of human tau protein: correlation with the tau pattern in brain and effects on tubulin polymerization. EMBO. J. 9, 4225-4230 (1990)

8. Goedert, M., Spillantini, M. G., Potier, M. C., Ulrich, J. \& Crowther, R. A. Cloning and sequencing of the CDNA encoding an isoform of microtubule-associated protein tau containing four tandem repeats: differential expression of tau protein mRNAs in human brain. EMBO. J. 8, 393-399 (1989).

9. Avila, J., Lucas, J. J., Perez, M. \& Hernandez, F. Role of tau protein in both physiological and pathological conditions. Physiol. Rev. 84, 361-384 (2004).

10. Perry, G. Solving the insoluble. J. Alzheimers. Dis. 9, 301-304 (2006). 3 Suppl.

11. Medina, M. \& Avila, J. Is tau a prion-like protein? J. Alzheimers. Dis. 40, S1-S3 (2014). Suppl 1.

12. Medina, M. \& Avila, J. The role of extracellular Tau in the spreading of neurofibrillary pathology. Front Cell Neurosci 8, 113 (2014).

13. Mirbaha, H., Holmes, B. B., Sanders, D. W., Bieschke, J. \& Diamond, M. I. Tau Trimers are the minimal propagation unit spontaneously internalized to seed intracellular aggregation. J. Biol. Chem. 290, 14893-14903 (2015).

14. Hyman, B. T. Tau propagation, different tau phenotypes, and prion-like properties of tau. Neuron. 82, 1189-1190 (2014).

15. Alonso, A. D., Beharry, C., Corbo, C. P. \& Cohen, L. S. Molecular mechanism of prion-like tau-induced neurodegeneration. Alzheimers. Dement. 12, 1090-1097 (2016).

16. Polydoro, M. et al. Soluble pathological tau in the entorhinal cortex leads to presynaptic deficits in an early Alzheimer's disease model. Acta. Neuropathol. 127, 257-270 (2014).

17. Ohm, T. G. The dentate gyrus in Alzheimer's disease 163, 723-740 (2007).

18. Aimone, J. B., Deng, W. \& Gage, F. H. Resolving new memories: a critical look at the dentate gyrus, adult neurogenesis, and pattern separation. Neuron. 70, 589-596 (2011).

19. Amaral D. G., Kondo H., Lavenex P. An analysis of entorhinal cortex projections to the dentate gyrus, hippocampus, and subiculum of the neonatal macaque monkey. J. Comp. Neurol. 522, 1485-1505 (2013).

20. Altman, J. Are new neurons formed in the brains of adult mammals? Science 135, 1127-1128 (1962)

21. Hollands, C., Bartolotti, N. \& Lazarov, O. Alzheimer's disease and hippocampal adult neurogenesis; exploring shared mechanisms. Front Neurosci 10, 178 (2016).

22. Gomez-Nicola, D. et al. Temporal dynamics of hippocampal neurogenesis in chronic neurodegeneration. Brain: a journal of neurology 137, 2312-2328 (2014). Pt 8.

23. Llorens-Martin, M. et al. GSK-3beta overexpression causes reversible alterations on postsynaptic densities and dendritic morphology of hippocampal granule neurons in vivo. Mol. Psychiatry. 18, 451-460 (2013).

24. Sahay, A. et al. Increasing adult hippocampal neurogenesis is sufficient to improve pattern separation. Nature. 472, 466-470 (2011).

25. McHugh, T. J. et al. Dentate gyrus NMDA receptors mediate rapid pattern separation in the hippocampal network. Science 317, 94-99 (2007).

26. Nakashiba, T. et al. Young dentate granule cells mediate pattern separation whereas old granule cells facilitate pattern completion. Cell 149, 188-201 (2012).

27. Ally, B. A., Hussey, E. P., Ko, P. C. \& Molitor, R. J. Pattern separation and pattern completion in Alzheimer's disease: evidence of rapid forgetting in amnestic mild cognitive impairment. Hippocampus. 23, 1246-1258 (2013). 
28. Wesnes, K. A., Annas, P., Basun, H., Edgar, C. \& Blennow, K. Performance on a pattern separation task by Alzheimer's patients shows possible links between disrupted dentate gyrus activity and apolipoprotein E in4 status and cerebrospinal fluid amyloid-beta42 levels. Alzheimers Res Ther 6, 20 (2014).

29. Perez, M., Valpuesta, J. M., Medina, M., Montejo de Garcini, E. \& Avila, J. Polymerization of tau into filaments in the presence of heparin: the minimal sequence required for tau-tau interaction. J. Neurochem. 67, 1183-1190 (1996).

30. Gomez-Ramos, A. et al. Characteristics and consequences of muscarinic receptor activation by tau protein. Eur. Neuropsychopharmacol. 19, 708-717 (2009).

31. Schuck, P. Size-distribution analysis of macromolecules by sedimentation velocity ultracentrifugation and lamm equation modeling. Biophys. J. 78, 1606-1619 (2000)

32. Laue, T. M. \& Stafford, W. F. 3rd Modern applications of analytical ultracentrifugation. Annu Rev Biophys Biomol Struct 28, 75-100 (1999).

33. Cole, J. L. Analysis of heterogeneous interactions. Methods. Enzymol. 384 212-232 (2004).

34. Jessberger, S. \& Gage, F. H. ZOOMING IN: a new high-resolution gene expression atlas of the brain. Mol. Syst. Biol. 3, 75 (2007).

35. Zhao, C., Teng, E. M., Summers, R. G. Jr., Ming, G. L. \& Gage, F. H. Distinc morphological stages of dentate granule neuron maturation in the adult mouse hippocampus. The Journal of neuroscience: the official journal of the Society for Neuroscience 26, 3-11 (2006).

36. Lugert, S. et al. Quiescent and active hippocampal neural stem cells with distinct morphologies respond selectively to physiological and pathological stimuli and aging. Cell. Stem. Cell. 6, 445-456 (2010).

37. Llorens-Martin, M. et al. Peripherally triggered and GSK-3beta-driven brain inflammation differentially skew adult hippocampal neurogenesis, behavioral pattern separation and microglial activation in response to ibuprofen. Translational psychiatry 4, e463 (2014).

38. Llorens-Martin, M., Torres-Aleman, I. \& Trejo, J. L. Pronounced individual variation in the response to the stimulatory action of exercise on immature hippocampal neurons. Hippocampus. 16, 480-490 (2006).

39. Llorens-Martin, M. \& Trejo, J. L. Mifepristone prevents stress-induced apoptosis in newborn neurons and increases AMPA receptor expression in the dentate gyrus of C57/BL6 mice. PLOS. ONE. 6, e28376 (2011).

40. Bolte, S. \& Cordelieres, F. P. A guided tour into subcellular colocalization analysis in light microscopy. J. Microsc. 224, 213-232 (2006). Pt 3.

41. Pallas-Bazarra, N., Kastanauskaite, A., Avila, J., DeFelipe, J. \& Llorens-Martin, M. GSK-3beta overexpression alters the dendritic spines of developmentally generated granule neurons in the mouse hippocampal dentate gyrus. Frontiers in neuroanatomy 11, 18 (2017).

42. Rodriguez, A., Ehlenberger, D. B., Dickstein, D. L., Hof, P. R. \& Wearne, S. L. Automated three-dimensional detection and shape classification of dendritic spines from fluorescence microscopy images. PLOS. ONE. 3, e1997 (2008).

43. Toni, N. et al. Neurons born in the adult dentate gyrus form functional synapses with target cells. Nat. Neurosci. 11, 901-907 (2008).

44. Bischofberger, J. Young and excitable: new neurons in memory networks. Nat. Neurosci. 10, 273-275 (2007)

45. van Praag, H. et al. Functional neurogenesis in the adult hippocampus. Nature. 415, 1030-1034 (2002)

46. Weible, A. P., Rowland, D. C., Pang, R. \& Kentros, C. Neural correlates of nove object and novel location recognition behavior in the mouse anterior cingulate cortex. J. Neurophysiol. 102, 2055-2068 (2009).

47. Bolz, L., Heigele, S. \& Bischofberger, J. Running improves pattern separation during novel object recognition. Neural. Plast. 1, 13 (2015).

48. Hernandez, F. \& Avila, J. Intra- and extracellular protein interactions with tau. Curr. Alzheimer. Res. 7, 670-676 (2010).

49. Ballatore, C., Lee, V. M. \& Trojanowski, J. Q. Tau-mediated neurodegeneration in Alzheimer's disease and related disorders. Nat. Rev. Neurosci. 8, 663-672 (2007).

50. Aronov, S., Aranda, G., Behar, L. \& Ginzburg, I. Axonal tau mRNA localization coincides with tau protein in living neuronal cells and depends on axonal targeting signal. The Journal of neuroscience: the official journal of the Society for Neuroscience 21, 6577-6587 (2001).

51. Dawson, H. N. et al. Inhibition of neuronal maturation in primary hippocampal neurons from tau deficient mice. J. Cell. Sci. 114, 1179-1187 (2001). Pt 6.

52. Dixit, R., Ross, J. L., Goldman, Y. E. \& Holzbaur, E. L. Differential regulation of dynein and kinesin motor proteins by tau. Science 319, 1086-1089 (2008).

53. Gotz, J. \& Nitsch, R. M. Compartmentalized tau hyperphosphorylation and increased levels of kinases in transgenic mice. Neuroreport. 12, 2007-2016 (2001).
54. Hernandez, F. \& Avila, J. Tauopathies. Cellular and molecular life sciences: CMLS 64, 2219-2233 (2007)

55. Frandemiche, M. L. et al. Activity-dependent tau protein translocation to excitatory synapse is disrupted by exposure to amyloid-beta oligomers. The Journal of neuroscience: the official journal of the Society for Neuroscience 34, 6084-6097 (2014)

56. Fuster-Matanzo, A., Llorens-Martin, M., Jurado-Arjona, J., Avila, J. \& Hernandez, F. Tau protein and adult hippocampal neurogenesis. Front Neurosci 6, 104 (2012).

57. Alvarez, A., Toro, R., Caceres, A. \& Maccioni, R. B. Inhibition of tau phosphor ylating protein kinase cdk5 prevents beta-amyloid-induced neuronal death. FEBS Lett. 459, 421-426 (1999).

58. Brion, J. P., Smith, C., Couck, A. M., Gallo, J. M. \& Anderton, B. H. Developmental changes in tau phosphorylation: fetal tau is transiently phosphorylated in a manner similar to paired helical filament-tau characteristic of Alzheimer's disease. J. Neurochem. 61, 2071-2080 (1993)

59. Drechsel, D. N., Hyman, A. A., Cobb, M. H. \& Kirschner, M. W. Modulation of the dynamic instability of tubulin assembly by the microtubule-associated protein tau. Mol. Biol. Cell. 3, 1141-1154 (1992).

60. Hanger, D. P., Anderton, B. H. \& Noble, W. Tau phosphorylation: the therapeutic challenge for neurodegenerative disease. Trends. Mol. Med. 15, 112-119 (2009).

61. Hong, X. P. et al. Essential role of tau phosphorylation in adult hippocampal neurogenesis. Hippocampus. 20, 1339-1349 (2010).

62. Andorfer, C. et al. Hyperphosphorylation and aggregation of tau in mice expressing normal human tau isoforms. J. Neurochem. 86, 582-590 (2003).

63. Arendt, T. Synaptic degeneration in Alzheimer's disease. Acta. Neuropathol. 118, 167-179 (2009)

64. Bretteville, A. \& Planel, E. Tau aggregates: toxic, inert, or protective species? J. Alzheimers. Dis. 14, 431-436 (2008).

65. Avila, J. Tau protein, the main component of paired helical filaments. J. Alzheimers. Dis. 9, 171-175 (2006). 3 Suppl.

66. Avila, J. Tau phosphorylation and aggregation in Alzheimer's disease pathology. FEBS Lett. 580, 2922-2927 (2006)

67. Bolos, M. et al. Direct evidence of internalization of tau by microglia In Vitro and In Vivo. J. Alzheimers. Dis. 50, 77-87 (2016).

68. Cameron, H. A., McEwen, B. S. \& Gould, E. Regulation of adult neurogenesis by excitatory input and NMDA receptor activation in the dentate gyrus. The Journal of neuroscience: the official journal of the Society for Neuroscience $\mathbf{1 5}$ 4687-4692 (1995)

69. Deshpande, A. et al. Retrograde monosynaptic tracing reveals the tempora evolution of inputs onto new neurons in the adult dentate gyrus and olfactory bulb. Proceedings of the National Academy of Sciences of the United States of America 110, E1152-E1161 (2013).

70. Llorens-Martin, M. et al. Selective alterations of neurons and circuits related to early memory loss in Alzheimer's disease. Front Neuroanat 8, 38 (2014).

71. Marin-Burgin, A., Mongiat, L. A., Pardi, M. B. \& Schinder, A. F. Unique processing during a period of high excitation/inhibition balance in adult-born neurons. Science 335, 1238-1242 (2012).

72. Vivar, C., Peterson, B. D. \& van Praag, H. Running rewires the neuronal network of adult-born dentate granule cells. Neuroimage. 131, $29-41$ (2016).

73. Arellano, J. I., Benavides-Piccione, R., Defelipe, J. \& Yuste, R. Ultrastructure of dendritic spines: correlation between synaptic and spine morphologies. Front Neurosci 1, 131-143 (2007).

74. Llorens-Martin, M., Rabano, A. \& Avila, J. The ever-changing morphology of hippocampal granule neurons in physiology and pathology. Front Neurosci $\mathbf{9}$ 526 (2015).

75. Khatoon, S., Grundke-lqbal, I. \& lqbal, K. Brain levels of microtubule-associated protein tau are elevated in Alzheimer's disease: a radioimmuno-slot-blot assay for nanograms of the protein. J. Neurochem. 59, 750-753 (1992).

76. Gomez de Barreda, E. et al. Tau-knockout mice show reduced GSK3-induced hippocampal degeneration and learning deficits. Neurobiol. Dis. 37, 622-629 (2010).

77. Guo, T., Noble, W. \& Hanger, D. P. Roles of tau protein in health and disease. Acta. Neuropathol. 133, 665-704 (2017).

78. Hanger, D. P. et al. Intracellular and extracellular roles for tau in neurodegenerative disease. J. Alzheimers. Dis. 40, S37-S45 (2014). Suppl 1.

79. Penazzi, L., Bakota, L. \& Brandt, R. Microtubule dynamics in neuronal devel opment, plasticity, and neurodegeneration. Int. Rev. Cell. Mol. Biol. 321, 89-169 (2016). 
80. Richetin, K. et al. Amplifying mitochondrial function rescues adult neurogenesis in a mouse model of Alzheimer's disease. Neurobiol. Dis. 102, 113-124 (2017).

81. Abisambra, J. F. et al. Tau accumulation activates the unfolded protein response by impairing endoplasmic reticulum-associated degradation. The Journal of neuroscience: the official journal of the Society for Neuroscience $\mathbf{3 3}$ 9498-9507 (2013).

82. Decker, J. M. et al. The Tau/A152T mutation, a risk factor for frontotemporalspectrum disorders, leads to NR2B receptor-mediated excitotoxicity. EMBO. Rep. 17, 552-569 (2016).

83. Takeda, S. et al. Neuronal uptake and propagation of a rare phosphorylated high-molecular-weight tau derived from Alzheimer's disease brain. Nat Commun 6, 8490 (2015).
84. Gomez-Ramos, A., Diaz-Hernandez, M., Cuadros, R., Hernandez, F. \& Avila, J. Extracellular tau is toxic to neuronal cells. FEBS Lett. 580, 4842-4850 (2006).

85. Gomez-Ramos, A., Diaz-Hernandez, M., Rubio, A., Miras-Portugal, M. T \& Avila, J. Extracellular tau promotes intracellular calcium increase through M1 and M3 muscarinic receptors in neuronal cells. Mol. Cell. Neurosci. 37, 673-681 (2008).

86. Simon, D., Hernandez, F. \& Avila, J. The involvement of cholinergic neurons in the spreading of tau pathology. Front Neurol 4, 74 (2013).

87. Avila, J., Simon, D., Diaz-Hernandez, M., Pintor, J. \& Hernandez, F. Sources of extracellular tau and its signaling. J. Alzheimers. Dis. 40, S7-S15 (2014). Suppl 1.

88. Lanzafame, A. A., Christopoulos, A. \& Mitchelson, F. Cellular signaling mechanisms for muscarinic acetylcholine receptors. Receptors. Channels. 9, 241-260 (2003). 